\title{
Por uma bibliografia sobre a teoria da dependência
}

\author{
THEOTÓNIO DOS SANTOS
}

A

SELEÇÃO DE LIVROS que se segue não tem a intenção de ser completa. Ela

serviu de base ao nosso texto sobre A teoria da dependência: 30 anos

depois, que faz parte do livro Os desafios do sistema mundial, organizado pelo Conselho de Ciências Sociais da Unesco para a América Latina em homenagem aos meus 60 anos.

É somente uma primeira abordagem que recolhe os principais textos que participaram de um debate amplo que dominou a imaginação sociológica de diferentes setores do pensamento social latino-americano, norte-americano, caribenho, africano, asiático e europeu. Poucas vezes um produto gerado no mundo periférico teve uma repercussão tão ampla.

No caso da teoria da dependência o que se universalizou foi uma maneira de ver os fenômenos, um princípio ordenador do pensamento, uma metodologia e um enfoque que desafia o mainstrean, o pensamento importado dos países centrais. Não se pode subestimar tal aspecto quando vemos alguns dos formuladores de uma teoria contestadora saltarem do barco a tempo de poder comprometer-se com o establishment científico, econômico e político-internacional.

Contudo, a Teoria das Relações Internacionais e a chamada Economia Política Internacional, entre outras disciplinas, reconhecem na teoria da dependência uma das alternativas de enfoque da economia política e das relações internacionais e um dos conceitos-chave dessas disciplinas.

O interessante é que, sendo os autores brasileiros parte principal da geração dessa teoria, seja esse país um dos menos representados na sua evolução posterior. É principalmente estranho o desconhecimento da teoria do sistema mundial que representa um dos mais belos frutos da teoria da dependência. Foi, por exemplo, extremamente interessante observar os cientistas sociais brasileiros descobrirem espantados a rica elaboração contida no livro de Giovanni Arrighi sobre o Longo século XXI, recentemente traduzido ao português.

Por tudo isto, consideramos útil divulgar esta bibliografia, ainda que incompleta, para ajudar a colocar nos seus devidos termos o lugar do pensamento social latino-americano, particularmente o brasileiro não-oficial, no pensamento mundial. 
ABEL, Christopher. Latin America, economic imperialism and the State: the political economy of the external connection from Independence to present. London, The Athlone Press, 1985.

ACKERMANN, Maria das Graças. Les entrepreneurs et le développement (étude d'un groupe d'industriels métallurgiques an Chili). Paris, Memoria presentada a l'École Pratique des Hautes Études, sept. 1970 [mimeo].

AGUILAR M., Alonso. Teoría y politica del desarrollo latinoamericano.

ALBERTI, Blas M. \& HOROWIEZ, Alejandro, La penetración imperialista en las ciencias sociales en América Latina. A propósito de André Gunder Frank y Theotônio dos Santos, Santiago, 1972.

ALSCHULER, Lawrence R. A sociological theory of Latin American underdevelopment. Comparative Studies, VI, 1973.

Satellization and stagnation in Latin America. International Studies Quatery, v. 20, n. 1, Mar. 1976.

ANDREFF, W. Les multinationales. Paris, La Découverte, 1987.

ANTOLA, Esko, Kehitysmaat kansain-vâlèsessä järtelmässa. In: Eeva-Liisa Milly-mäki \& Lamá Honko (ed.). Kehitsmaatiedon Perusteet. Turku, Turun hehitymaatiedon tyorijmà, Turku, 1976, p. 127-128.

AMIN, Samir. Accumulation on a world scale: A critique of the theory of underdevelopment. New York, Monthly Review Press, 1974.

APTER, David E. Rethinking development modernization, dependency and postmodern politics. London, Sage Publications, 1990.

BACHA, Claire Savit. A dependncia nas relações internacionais: uma introdução à experiencia brasileira. Rio de Janeiro, 1971. Dissetação (mestrado). IUPERJ.

BAGÚ, Sergio. Dependencia y subdesarrolo en América Latina, comentarios. Problemas del desarrollo, México, UNAM, n. 4, 1970.

BAMBIRRA, Vania. Integración monopólia mundial e industrialización: sus contradicciones. Santiago, Sociedad y Desarrollo, v. I, n. 1, 1972. Teoria de la dependencia: una anticritica. México, Era, 1978.

. El capitalismo dependiente en América Latina. México, Siglo XXI,

México.

La Revolución Cubana: una reinterpretación. México, Ed. Nuestro

Tiempo, 1974.

(introducción y compilación). Diez años de experiencia insurreccional en América Latina.

BEAUD, Michel. Sur la connaissance de l'économie mondiale. Paris, 1990 [mimeo].

BITAR, Sergio. Inversión extranjera en la industria manufacturera de Chile.

BIZELLI, Edimilson. La política norteamericana para Améirca Latina. Caracas, Economía $y$ Ciencias Sociales, $\mathrm{n}^{\circ}$ extraordinario en acuerdo con el CESO, Dic. 1973. 
BLOMSTROM, Magnus. Development theory in trasition, the dependency debate \& beyond: Third World responses. Londres, Zed Books, 1984.

BODENHEIMER, Suzzane. Dependency and imperialism. Politics and Society, n.5, maio 1970.

Dependency and Imperialism: The roots of Latin American underdevelopment. NACLA Newsletter, 1970.

BRAND, Willy. Commission report, North-South; a programme for survival. London/ Sidney, Pan Books, 1980.

Commission report, common crisis North-South; cooperation for world recovery. London/Sidney, Pan Books, 1983.

Global challenge, from crisis to cooperation; breaking the North-South stalematc. Report of the Socialist International Committee on Economic Policy. London/Sidney, Pan Books, 1985.

BRAUDEL, Fernand. Économie-monde. Civilisation matérielle, économic et capitalisme, v. 3: Le temps du monde. Paris, Armand Colin, 1979.

BRIONES, Álvaro. Economía y ciencias sociales, sobre empresas transnacionales y dependencia tecnológica. Caracas, Los conglomerados transnacionales, la tecnología y el mercado de bienes intermedios, $\mathrm{n}^{\circ}$ extraordinario en acuerdo con el CESO, Dic. 1973.

CAMACHO, Daniel. Debates sobre la teoria da la dependencia y la sociologia Latinoamericana. San José, EDUCA, 1979.

CAMPOS, M.N. Transferencia de tecnologia, dependencia del exterior y desarrollo económico.

CARDOSO, Fernando Henrique. Teoría de la dependencia o análisis de situaciones concretas de dependencia? Revista Latinoamericana de Ciencia Política, I, dic. 1971.

Imperialismo e dependencia, 1972 [mimeo].

Estado y sociedad.

CARMONA DE LA PEÑA, Fernando. Dependencia y cambios estructurales.

CASTRO, Josué. Geopolítica da fome, 1951.

CASTRO, Fidel. The world economic and social crisis. Delhi, People's Publishing House, 1983.

CECEÑA CERVANTES, José Luis. Superexplotación, dependencia y desarrollo.

CINTA, Ricardo. Burguesía nacional y desarrollo. In: El perfil de México en 1980.

CHILCOTE, Ronald H. A critical synthesis of the dependency literature. Latin American Perspectives, I, Primavera 1974.

Theories of development and underdevelopment. Boulder/London, Ed. Westview Press, 1984.

Dependency and marxism; toward a revolution of the debate. Boulder/London, Ed. Westview Press, 1982. 
CLUB OF ROME. The limits to growth. New York, Universe Books, 1972.

CÓRDOBA, Armando y SILVA MICHELENA, Héctor. Aspectos teóricos del subdesarrollo. Armando, El capitalismo subdesarrollado de A.G. Frank.

CÓRDOVA, Arnaldo. La politica de masas del cardenismo.

CUEVA, Agustín. El desarrollo del capitalismo en América Latina. México, Siglo XXI, 1978.

Problemas y perspectivas de la teoria de la dependencia. México, CELA, UNAM.

DAVYDOV, Vladimir. Nueva ronda de debates acerca de la dependencia. Moscou, América Latina, n. 11, 1984.

Que es la teoria de la dependencia? Moscou, América Latina, n. 3, 1985 e n. 12, 1986.

DOLPHUS, Olivier. Le système monde. L'Information Géographique, n. 54, p. 45-52.

DOS SANTOS, Theotônio. La crisis Norte-Americana y América Latina. Santiago, Ed. PLA, 1970.

Imperialismo e dependência na América Latina. In: F. Bonilla \& Girling (ed.). Structures of dependency. Stanford, CA, Institute of Political, 1973.

Imperialismo y corporaciones multinationales. Santiago, Ed. PLA, 1973.

- Imperialismo y dependencia. México, Era, 1978.

. Osfundamentos teóricos do governo Fernando Henrique Cardoso. Porto Alegre, Cienciais \& Letras, 1996.

América Latina: democracia e ajuste estrutural. Porto Alegre, Anos 90, 1996-b.

DREIFUSS, René. A internacional capitalista: estratégias e táticas do empresariado transnacional - 1918 a 1986. Rio de Janeiro, Ed. Espaço e Tempo, 1987.

DUNN, C. Chase. The effects of international economic dependence on development and inequality: a cross national study. American Sociological Review, XL, dez. 1975.

DURAND P., Víctor Manuel. México: dependencia o independencia en 1980. El Perfil de México en 1980.

DUVALL, Raymund et al. A formal model of 'dependencia' theory: structure, measurement and some preliminary data. Edimburgh, IPSA Congress of August 1621, 1976.

FAJNZYLBER, Fernando. Sistema industrial en Brasil, 1970. Sistema industrial y exportación de manufacturas.

FARIA, Vilmar E. Dépendence et idéologie des dirigeants industriels brésiliens. Paris, Sociologie du Travail, n. 3, jul./sept. 1971. [Faria escribió también una monografía todavía inédita sobre el mismo tema]. 
FAUSTO, Ayrton. La nueva situación de dependencia y el análisis sociopolítico de Theotônio dos Santos. Santiago, Revista Latinoamericana de Ciencias Sociales, n.12,1971 .

FERNANDES, Florestan. Patrones de dominación externa en América Latina. Revista Mexicana de Sociología, v. XXXII, n.6, nov./dic. 1970.

FRANK, André Gunder. El desarollo del subdesarollo: un ensayo autobiográfico. Ed. Nueva Sociedad, 1991.

World accumulation, 1492-1789, Monthly Review, 1978.

1978.

Dependent accumulation and Underdevelopment, Monthly Review,

Crisis in the world economy. New York, Holmes \& Meier, 1980.

Crisis in the Third World. New York, Holmes \& Meier, 1981.

A theoretical introduction to 5000 years of world system history. Review, Binghamton, v. XIII, n. 2, p 155-248, Primavera, 1990.

FURTADO, Celso. O mito do desenvolvimento economico.

FUKUYAMA, Francis. The end of history and the last man, cap. 9, 1992.

GALTUNG, Johan. A structural theory of imperialism. Journal of Peace Research, n. 2, p. 81-117, 1971.

GARCÍA, Antonio. Atraso y dependencia en América Latina. Hacia una teoría latinoamericana de las ciencias sociales del desarrolllo.

GIRVAN, Norman. The development of dependency economics in the Caribbean and Latin America. Review and Comparison, Social and Economic Studies, XXII, mar. 1973.

GONZÁLES, Estanislao. Venezuela: nueva política petrolera y dependencia. Economía y Ciencias Sociales. Review and Comparison, Social and Economic Studies, XXII, mar. 1973.

GRACIARENA, Jorge. La dinámica del capitalismo del subdesarrollo en América Latina. México, Foro Internacional, XIII, abr./jun. 1973.

GROU, Pierre. Atlas mondial des multinationales e l'espace des multinationales. Paris, Réclus-La Documentation Française, 1990.

HASENBALG, G.; BRIGADÃO C. \& LEITE COSTA, F.J. O sector financiero no Brasil: aspectos históricos.

HERRERA, Amilcar. Catastrophe or new society? A Latin American world model. Ottawa, IDRC, 1976.

Las nuevas tecnologias y el futuro de América Latina.

Development theory and the three worlds, 1990.

HETTNE, Bjorn \& BLOMSTRÖM, Magnus. Development theory in trasition. The dependency debate \& beyond; Third World reponses. London, Zed Books, 1984. Edição em espanhol por Siglo XXI, 1990. 
HINKELAMMERT, Franz. El subdesarrollo latinoamericano. Un caso de desarrollo capitalista.

La teoría clásica del imperialismo, el subdesarrollo y la acumulación socialista. Santiago, Cuadernos de la Realidad Nacional, n.4, jun. 1970.

Teoría de la dialéctica del desarrollo desigual. Santiago, Cuadernos de la Realidad Nacional, n.6, 1970.

HUNT, Economic Theories of Development, 1989.

HVEEM, Helge. The global dominance system. Notes on a theory of global political economy. Journal of Peace Research, n. 4, p. 319-340, 1973.

IMEMO - Institute of World Economy and International Relations of the Science Academy. Developing countries: regularities. Tendencies and perspectives. Editado na Russia em 1978.

IANNI, Octavio. Imperialismo y cultura de la violencia en América Latina.

$$
\text { Sociología del imperialismo. }
$$

IANNI, Octavio La sociología de la dependencia en América Latina. Asunción, Revista Paraguaya de Sociología, v. I, n. 21, mayo-ago. 1971.

KAHL, Joseph A. Modernization exploitation and dependency in Latin America. New Jersey, New Brunswick, 1976.

KAY, Cristobal. Latin American theories of development and underdevelopment, 1989.

KAUFFMAN, Robert. A preliminary test of the theory of dependency. Comparative Politics, VII, abr. 1975.

LABASTIDA, J. Los grupos dominantes frente a las alternativas de cambio. El perfil de México en 1980.

LALL, Sanjaya. Is dependence a useful concept in analysing underdevelopment? World Development, III, nov. 1975.

LARRAÍN. Theories of development, 1989.

LE ROY, Cis et al. Toward a resolution of the weakness of dependency theory. Riverside, University of California, Collective Paper of Graduate Students, 1973.

LECHMAN. Democracy and development in Latin America, 1990.

LEBEDINSKY, Mauricio. Del subdesarrollo al desarrollo. América Latina en la encrucijada de la década del setenta.

LEIVA, J.L. El sector externo, los grupos sociales y las politicas económicas en Chile (18301940), CESO [mimeo].

LEONTIEF, W. Les multinationales. Paris, La Découverte, 1987.

LESSA, C. \& VASCONI, T. Hacia una critica de las interpretaciones del desarrollo latinoamericano. Caracas, Universidad Central de Venezuela, 1969.

LEWIS, Colin M. Latin America, economic imperialism and the State; the political economy of the external connection from Independence to present. London, The Athlone Press, Londres, 1985. 
LUCIANO, Martins. Politique et développement économique: structures de pouvoir et système de décisions au Brésil. Paris, Anthropos.

MAIDÁNIK, Kiva. El proceso revolucionário de America Latina visto desde la URSS. Santo Domingo, Republica Dominicana, Editora Taller, C. por A., 1982.

MALAVÉ-MATTA, Héctor. Dialéctica del subdesarrolo y dependencia. México, Problemas del Desarrollo, ago./oct. 1972.

MANLEY, Michael. Global challenge, from crisis to cooperation; breaking the NorthSouth stalematc. Report of the Socialist International Committee on Economic Policy. London/Sidney, Pan Books, 1985.

MARINI, Ruy Mauro. Dialéctica de la dependencia.

MARTZ, John. Political science and Latin American studies: a discipline in search of a region. Latin American Research Review, VI, Primavera, 1971.

MAZA ZAVALA, D.F. Los mecanismos de la dependencia.

MEEROPOL, Michael. Towards a political economy analysis of underdevelopment. Review of Radical Economy, IV, 1972.

MICHELET, C.A. Le capitalisme mondial. Paris, P.U.F., 1985.

MORENO, José. CEPAL, reformismo e imperialismo.

MUÑOZ, Heraldo. El análise de la teoria de la dependencia en los centros: ejemplos de EEUU. Estudios Internacionales, jan./mar. 1979.

Cambio y continuidad en el debate sobre la dependencia y el imperialismo. Estudios Internacionales, out./dez. 1978.

From dependency to development-strategies to overcome underdevelopment and inequality. Boulder, Colorado, Editora Westview Press, 1982.

MURGA, Antonio. Dependency: a Latin American view. NACLA Newsletter, IV, febrero de 1971.

NGO MAN LAN. Un real growth, critical studies in Asian development, 2 v. Delhi, Hindustan Publishing Corporation, 1984.

O'BRIEN, Phillip. A critique of Latin American theories of dependence. In: Oxgal et al., Beyond the sociology of development, Londres, 1975.

O DESAFIO AO SUL. Relatório da Comissão Sul. Ed. Afrontamento, 1990.

OLOF PALME. Commission report, common security; a program for disarmament. London/Sidney, Pan Books, 1982.

OMAN, Charles. The postwar evolution of development thinking. Paris, OECD Development Center, 1991.

PEET, Richard. Global capitalism - theories of societal development. London/New York, Routledge, 1991.

PERALTA, Ramos M. Etapas de acumulación de capital y lucha de clases en la Argentina, 1930-1970. 
PEREIRA, Y. E. Endeudamiento exterior.

PINTO Anibal. El modelo de desarrollo reciente en América Latina. México, El Trimestre Económico, v. 37, n. 150, 1970.

Notas sobre desarrollo, subdesarrollo y dependencia. México, El Trimestre Económico, v. 39, n. 154, 1972.

El sistema centro-periferia 20 años después. In: International Economics. Ensayos en honor de Raúl Prebisch, Estados Unidos, Ed. L.E.D. Marco, Academic Press, 1972.

PIZARRO, Roberto \& CAPUTTO, Orlando. Imperialismo, dependencia y relaciones económicas internacionales. Santiago, Ed. PLA, 1970.

Chile. mi.

Desarrolllo y capital extranjero: las nuevas formas del imperialismo en

PNUD. Human Development Report, 1991.

PORTANTIERO, Juan Carlos. O marxismo Latinoamericano. In: History of marxism, dirigida por Eric J. Hobsbawn.

PREBISCH, Raul. Capitalismo periférico, crisis y transformación. México, Fondo de Cultura Económica, 1981.

QUIJANO, Aníbal. Nationalism and capitalism in Perú: a study in neo imperialism. Monthly Review, v. 23, n. 3, jul./ago. 1971.

RAMOS, Sergio. Chile: una economía en transición? Santiago, Ed. PLA, 1970.

REYNA, J.L. Movilización o partipación politica: discusión de algunas hipótesis para el caso mexicano [Además de este trabajo ver su tesis de doctorado].

RODRIQUEZ, Gustavo O. De la Cepal a la teoria de la dependencia - un esquema descriptivo. Cochabamba, IESE, 1979.

RODRIQUEZ, Octavio. Informe sobre las criticas a la conception de la Cepal. México, Secretaria de la Presidencia, 1974.

ROSTOV, W.W. Etapas do crescimento econômico. Zahar, 1961.

ROSTOV, W.W.. The world economy: history and prospects. Austin, University of Texas Press, 1978.

Theories of economic growth from David Hume to the present with a perspective in the next century, 1994.

RUSSET, Bruce. An empirical assessement of global inequalities and dependence. Seminar THEORIES OF DOMINANCE AND DEPENDENCY STRUCTURES. Oslo, 13-14 March 1975. Proceedings, v. II, Oslo, PRIO, 1975.

SCHMIDT, Benicio Viera. Um teste de duas estratégias politicas: a dependência e a autonomia. Belo Horizonte, 1970. Dissertação (mestrado).

SEMO, Enrique. La crisis actual del capitalismo. México, Ed. de Cultura Popular, 1975.

SENTZES, Tamas. The political economy of underdevelopment. Budapest, Akadémiari Kiadó, 1971. 
SEPÚLVEDA, Cristian. Desarrollo económico en Chile. Santiago, Cuadernos del CESO, 1973 [mimeo].

SO, Alvin Y. Social change and development, modernization, dependency and world system theories. London, Sage Library of Social Research, 1990.

SONNTANG, Heintz R. Duda/certeza/crisis. La evolución de las ciencias sociales de América Latina. Caracas, Unesco-Editorial Nueva Sociedad, 1989.

SUNKEL, Oswaldo. Política nacional de desarrollo y dependencia externa. Santiago, Revista de Estudios Internacionales, v. I, n. 1, mayo 1967.

Capitalismo transnacional e desintegração nacional na América Latina. Social and Economic Studies, University of West Indies, 22-1, 1973.

STAVENHAGEN, Rodolfo. The future of Latin America: between underdevelopment and revolution. Latin American Perspectives, v. I, n.1, 1974.

Cómo descolonizar las ciencias sociales? Sociología y subdesarrollo.

THE GLOBAL 2000. Report to the president of the US. Washington, Government Printing Office, 1980.

THE JOURNAL OF INTERNACIONAL STUDIES. Edição especial dedicada à Teoria da dependência. Fev. 1973.

TIMBERGEN, Jean (coord.). Reshaping the internacional order, Rio. Dutton/New York, Report to the Club of Rome, 1976.

TODARO, M.P. Economic development in the Third World. London, Longman, 1977.

TUOMI, Helena. Denpendency models in Western development research. In: Dependency \& Latin American development. SEMINAR ON LATIN AMERICA: A REPORT, EEVA-LUISA MYLLIMÄKÍ E BRETT DELLINGER. Ed. Rauhaan Tutkien, Finish Peace Research Association, 1977.

UNITED NATIONS. Center on Transnational Corporations. World investment report. New York, 1992, 1993, 1994, 1995, 1996.

VAITSOS, C. Comercialización de tecnología en el Pacto Andino.

VASCONI, T., Dependencia y superestructura y otros ensayos. E colaboración con Inés Recca, Modernización y crisis en la Universidad Latinoamericana.

VÄYRINEN, Raimo. The role of transnational corporations in international trade. Tampere Peace Research Institute, Occasional Papers, n. 3, 1976.

VILLA, M. Las bases del Estado mexicano y su problemática actual. El perfil de México en 1980 .

VUSKOVIC, P. Distribución del ingreso y opciones de desarrollo. In: CEREN. Santiago, Universidad Católica de Chile, 1970.

WALLERSTEIN, Immanuel. The capitalist world economy. Maison des Sciences de I'Homme, 1979. 1984.

The politics of the world economy. Maison des Sciences de I'Homme, 

v. III, 1989.

Modern world system. New York, Academic, v. I, 1974; v. II, 1980;

WEFFORT, F.C. Clases populares y desarrollo social. ILPES, feb. 1968.

WIGNAJARA, Ganeshan. The postwar evolution of development thinking. Paris, OECD Development Center, 1991.

Theotônio dos Santos é professor da Universidade Federal Fluminense (RJ). 